\title{
EXPERIMENTAL AND NUMERICAL ANALYSIS OF POST-BUCKLING DEFORMATION STATES OF INTEGRALLY STIFFENED THIN-WALLED COMPONENTS OF LOAD-BEARING AIRCRAFT STRUCTURES
}

\author{
Tomasz Kopecki, Łukasz Święch \\ Rzeszów University of Technology, Faculty of Mechanical Engineering and Aeronautics, Rzeszów, Poland \\ e-mail: tkopecki@prz.edu.pl; l_swiech@prz.edu.pl
}

\begin{abstract}
The study presents results of experiments and numerical analyses concerning thin-walled shells used as components of aircraft structures. The solution, which is proposed here, consists in stiffening such elements by means of integral ribs. A comparative analysis has been carried out between the suggested design solution and the reference structure. In the experimental part of the study, an optical scanner with digital image correlation has been used. Nonlinear numerical analyses have been carried out with the use of software based on the finite element method.
\end{abstract}

Keywords: bifurcation, experiment, finite elements, thin-walled structures

\section{Introduction}

One of the fundamental requirements faced typically by design engineers in the process of developing modern airframe solutions is the necessity to ensure adequate strength of the load-bearing structure while keeping its weight at the minimum. Regardless of long-standing experience demonstrated by design teams, support provided by various optimisation theories, rapid developments in material engineering and new techniques of shaping structural elements, the practice of aircraft operation forces permanent intensification of research aimed at developing aviation structures with more favourable payload-to-empty weight ratio.

In the modern aircraft engineering, two basic trends can be observed in the approach to the aircraft load-bearing structure design. The first option prefers the use of composites, based mainly on carbon and aramid fibres, for an increasingly widening range of applications. The second attitude is based on the use of metals which, in combination with currently available advanced technologies, offer virtually unlimited possibilities in the area of shaping structural elements (Kopecki and Mazurek, 2013, 2014).

The use of composite materials, despite unquestionable benefits such as very favourable strength properties and a relatively low mass, is connected with a serious risk caused by insufficient knowledge of the physical phenomena occurring in materials of this type, which can result in unsatisfactory fatigue strength and, consequently, reduced durability, endurance, and serviceability. Hence, it seems that further research should focus on the use of metal materials, since their properties are far better known and proven by extensive operational experience.

The type of the structure most often used in aviation industry is the structure defined as semi-monocoque with the skeleton consisting of longitudinal elements (longerons) and transverse components (formers, ribs). The joints of skeleton components are treated as ideal hinges, thus the skeleton itself is a mechanism. The freedom in the change of the angles between formers and longerons is normally removed by the connection of the skeleton with the skin. It is normally assumed that the only possible interaction between the skin and the skeleton elements are tangential stresses, hence the only function of the skin is to work under shear forces, whereas the longitudinal forces are transferred by longerons (Brzoska, 1956; Niu, 1988). 
Because it is necessary to limit the weight, in the case of certain shells used in the aviation industry, stability loss is permitted within acceptable loads as long as such loss is of a local nature, i.e. when only elements of the skin between the skeleton components are subject to buckling. Even though it is acceptable, this phenomenon causes a significant redistribution of the states of stress and structure stiffness (Arborcz, 1985; Marcinkowski, 1999). Therefore, it becomes necessary to examine the operation of the structure in advanced post-critical deformation states (Kopecki, 2010; Kopecki and Mazurek, 2014).

Due to the necessity to prevent the global loss of stability, it is frequently indispensable to introduce additional stiffening elements, most often in form of longerons of relatively small cross-sections. In traditional solutions, such elements are joined together with the outer layer of the aircraft body by riveted seams, but this approach complicates the technological process, causes an increase in the aircraft mass and generates discontinuity in the structure of the material, which reduces its operating effectiveness and often leads to formation of fatigue cracks.

As a result of recently observed progress in material removal processes supported with numerical control as well as developments in 3D printing technology, an alternative solution to be applied for structures of this type involves the use of stiffening elements, which are integral with the skin and which make it possible to more effectively take advantage of the material properties (Fig. 1).
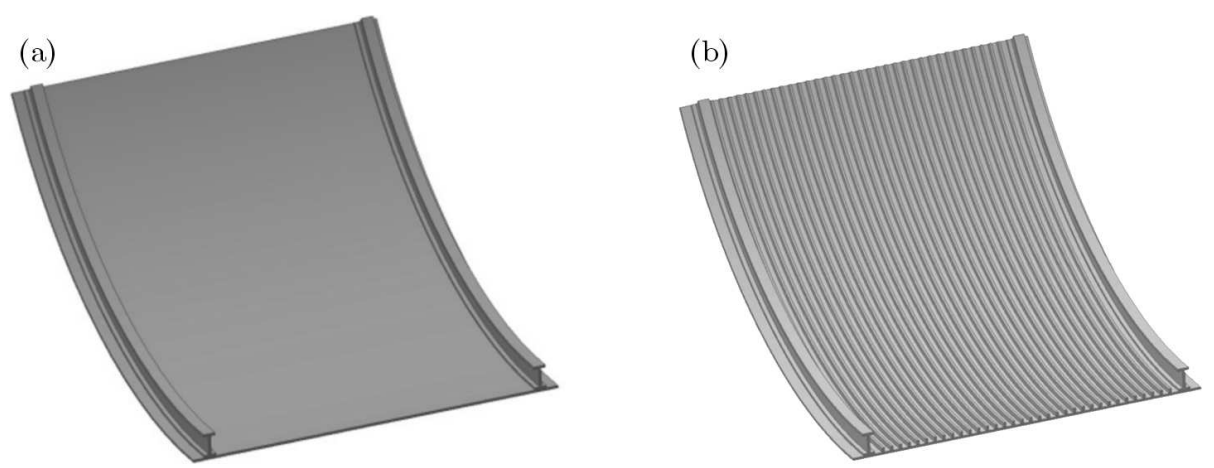

Fig. 1. Comparison of structural solutions: (a) conventional skin - smooth, (b) suggested solution - skin integrally ribbed

Although sysytems of this type are applied in aircraft structures, there are few available scientific studies of their properties, particularly related to the length of their service time, probably due to the confidentiality policies maintained by companies engaging their potential in long-term and costly research. Therefore, given the risk related to the use of composite structures whose properties have not been thoroughly investigated, it seems that there is a need to carry out research focusing on integrally reinforced thin-walled skins, and aiming at identifying their mechanical properties in advanced conditions of post-critical deformation states.

\section{Subject and scope of study}

The material examined during the study consists of panels made of model material, i.e. polycarbonate known by its trademarked name "macrolon" and having known physical characteristics. The structures which are investigated constitute reproductions of a fragment of an aircraft skin structure exposed to tangential stresses.

The study is designed as a comparative analysis of a smooth reference panel and an integrally stiffened panel. Both panels are of the same dimensions (Fig. 2). The smooth panel has thickness of $2 \mathrm{~mm}$ and mass of $206 \mathrm{~g}$. The ribbed structure is a $1 \mathrm{~mm}$ thick panel strengthened with 15 ribs, 
with the width of $2.5 \mathrm{~mm}$ and the height of $8 \mathrm{~mm}$. Its weight is $181 \mathrm{~g}$, which is $12 \%$ less than the reference panel.

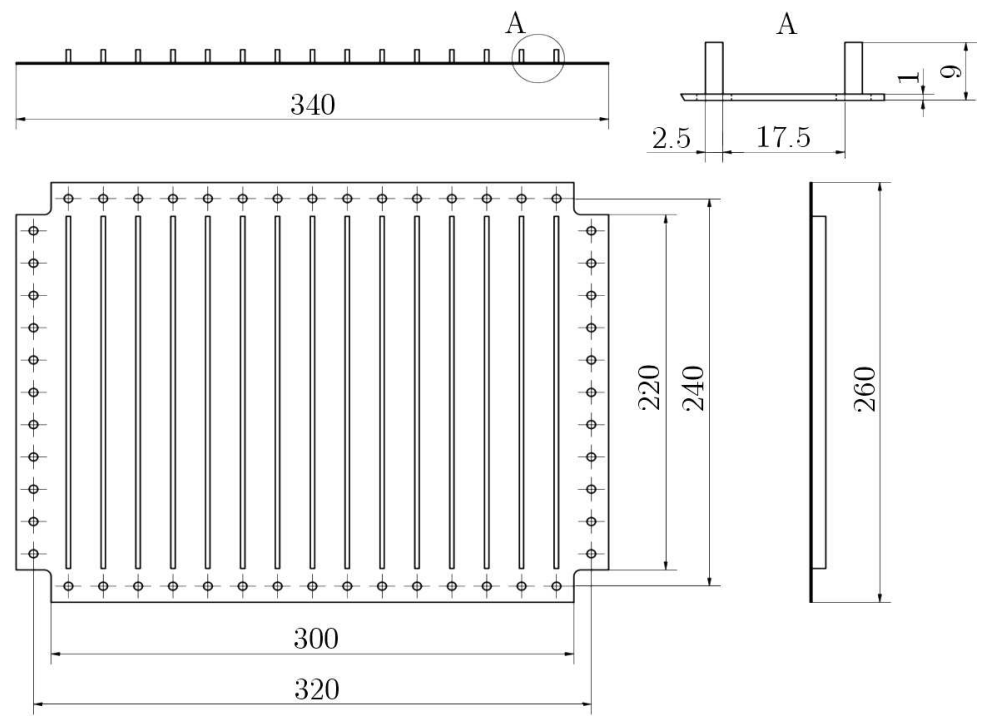

Fig. 2. Geometry of the investigated structure

The structure is made with the use of numerically controlled gate milling plotter (Fig. 3). To ensure adequate precision, a special vacuum table has been designed and constructed in order to immobilize the treated material without the need to use additional fasteners (Fig. 3).

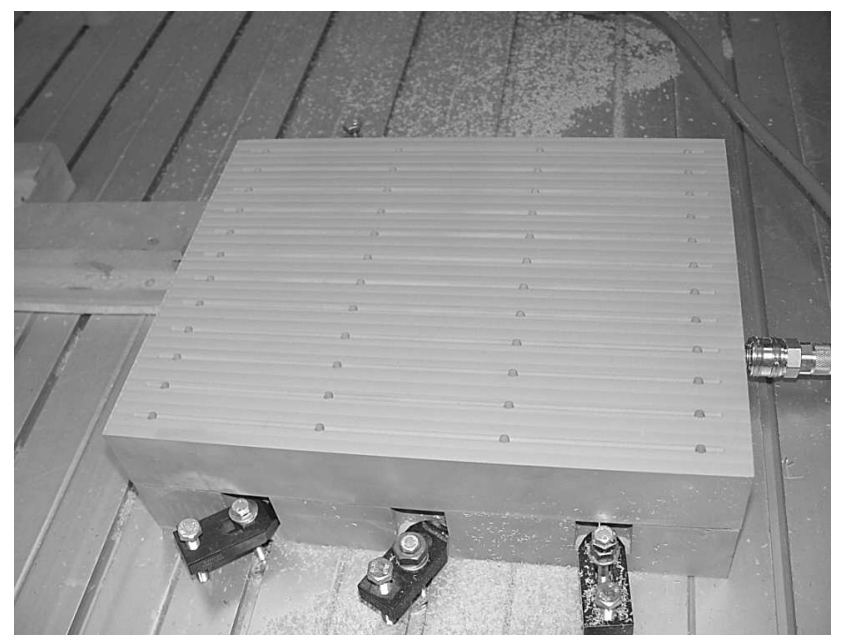

Fig. 3. Vacuum table for fastening the panels during treatment

Manufacturing deviations are determined with the use of ATOS white light scanner in combination with the digital photogrammetry system, TRITOP manufactured by GOM GmbH. Measurements of manufacturing deviations have shown that the examined ribbed panel contain thickness deviations below $0.1 \mathrm{~mm}$.

The tests have been designed to determine the impact of the integral strengthening elements on the nature of deformation in the ribbed panel by comparing the behaviour of both investigated structures in the conditions of post-critical loads. The results of the experimental tests are taken into account in designing methods for creating adequate numerical models of integrally stiffened panels in accordance with the finite element method (FEM). 


\section{Experimental tests}

The experiment has been carried out with the use of specially designed and constructed system for fastening the model. The system consists of a frame made of highly rigid flat bars, joined in corners in articulated manner with assembly pins (Fig. 4). Each of the panel edges has been fastened between two flat bars by means of bolt connections whose force of pressure is controlled during assembly. Such a solution, with sufficient rigidity of the flat bars, prevents the panel edges from rotation, thus the fastening conditions allow for nearly perfect stability.
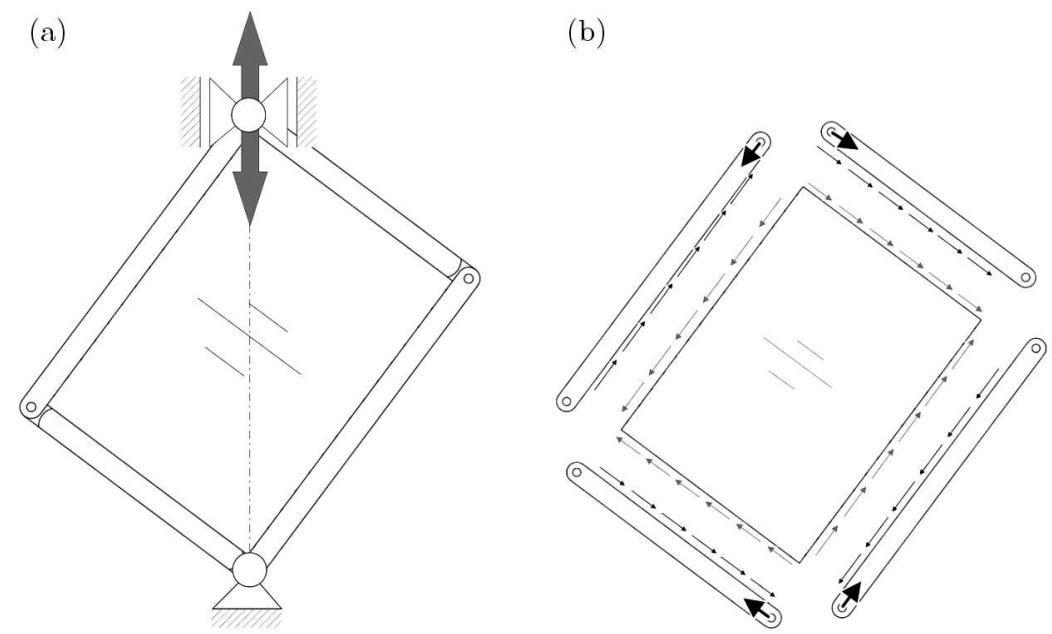

Fig. 4. Diagram of the panel fastening and load

A strength testing machine has been used as the loading system. The lower and upper frame nodes have been connected with the base and the cross-beam of the machine respectively (Fig. 5). As a result the model has been loaded in the form of tangential yield (Fig. 4).

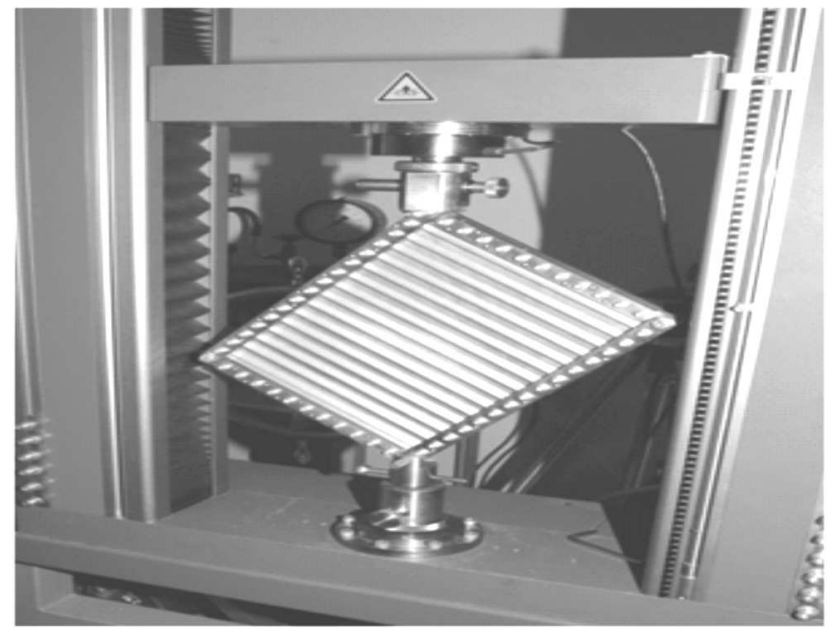

Fig. 5. Station located in the strength testing machine

Thanks to the use of a digital control and recording system it is possible to determine, in real time, both the current load value and the position of the loading cross-beam. Additionally, the station is calibrated in order to reduce measurement errors resulting from the limited stiffness of the fasteners. The calibration has been carried out with ARAMIS optical scanner employing the method of Digital Image Correlation (DIC).

During the first phase of the experiment, an analysis has been carried out to determine post-critical deformation in the smooth 'reference' panel. As a result the information in form of a 
displacement field versus the load value has been obtained. This method of recording the results makes it possible to create a representative equilibrium path of the system as a relationship between the load and any component of displacement in any point of the model.

Due to the loss in stability, the reference panel got a new form of the equilibrium with three folds: the dominant one with the highest deflection along the diagonal of the panel, and two minor ones distributed symmetrically against the diagonal (Fig. 7a). This form does not change regardless of the applied load value. It is assumed that the representative equilibrium path of the panel are the relationships between the displacement of the panel central point corresponding to the maximum deflection value and the value of the load force (Fig. 6a).
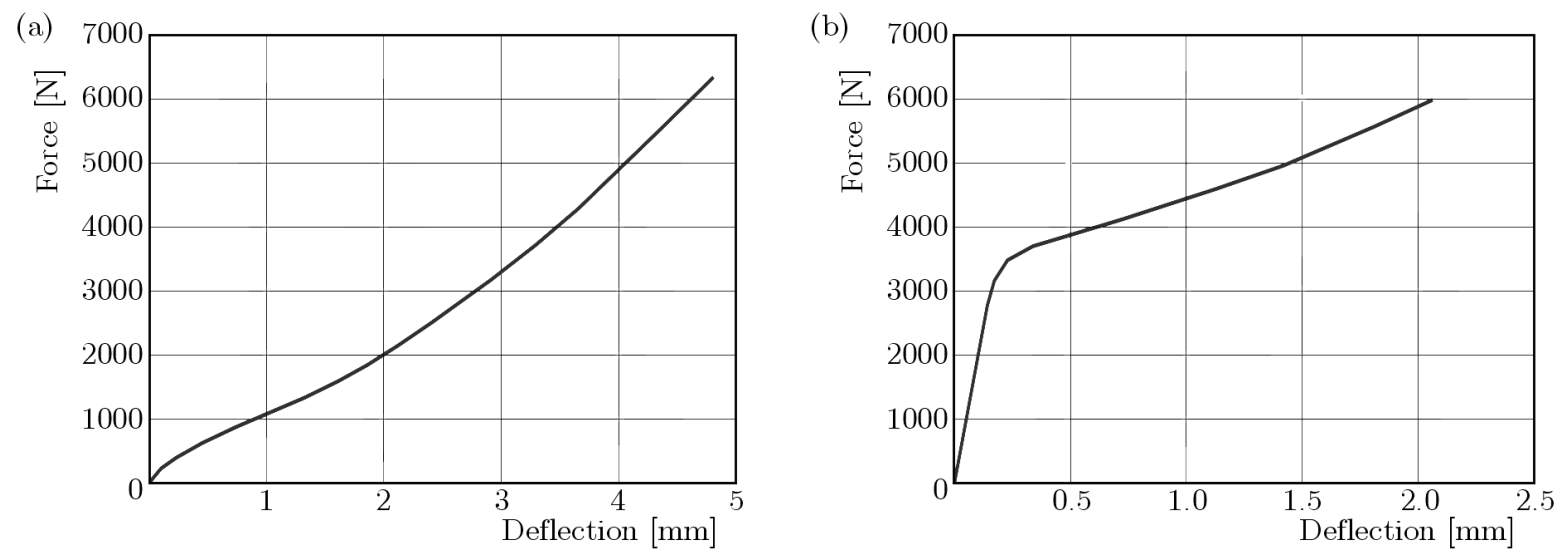

Fig. 6. Equilibrium paths determined by DIC method: (a) smooth panel, (b) ribbed panel
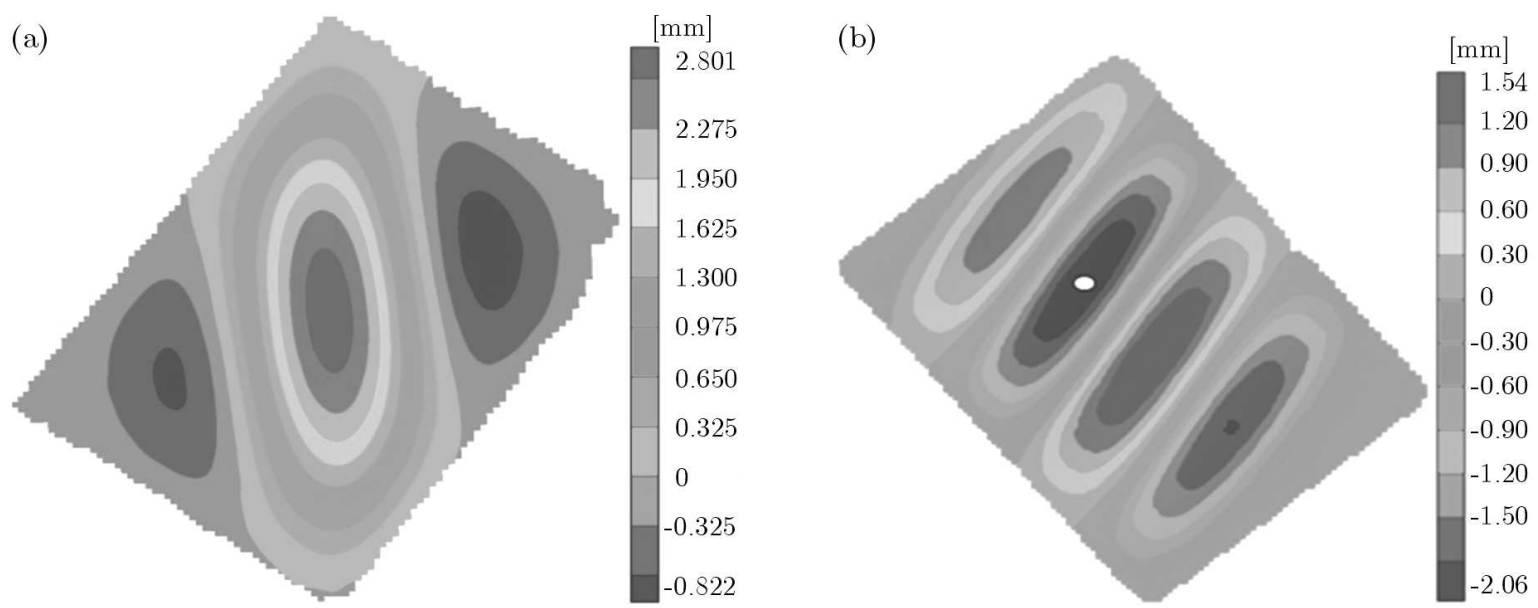

Fig. 7. Distribution of normal displacements: (a) smooth panel, (b) integrally ribbed panel (the location corresponding to the greatest displacement is marked with the white point)

The next phase of the experiment involved testing of the integrally stiffened panel. In this case, the nature of stability loss turned out to be considerably different than that observed for the reference panel. The form of the post-critical deformation showed four folds of a comparable deflection rate, oriented at the angle of about $30^{\circ}$ against the direction of the shorter side of the panel (Fig. 7b). Also in this case, the deformation form does not undergo any change with further increase in the load.

Due to the fact that the forms of post-critical deformations turned out to be different, the determination of the representative equilibrium paths illustrating the displacements of the point with the same coordinates is irrelevant. It is assumed that the changes in the state of the ribbed panel structure would be represented by the point undergoing the largest displacement 
located on the fold with the biggest deflection (Fig. 6b). Therefore, ultimately the representative equilibrium paths reflect the relations between the load and the maximum deflection of the panel, which makes it possible to compare them. It should be emphasized that the first visible effects of the loss in stability of the integrally ribbed plate appears at the load $75 \%$ higher than is the case of the smooth panel.

\section{Numerical analysis}

Numerically supported processes of designing aircraft structures even in their early stages include the phase of numerical analysis. This makes it possible to eliminate possible erroneous engineering assumptions leading to reduced operational durability of the designed structures (Niu, 1988).

In the case of thin-walled skins, it is necessary to develop adequate numerical models which make it possible to map the form of post-critical deformations and determine the levels of critical loads. Instruments most frequently used for this purpose include programs based on the finite element method, which makes it possible to conduct non-linear analyses. As for thin-walled models of aircraft structures, acceptability of stability loss is connected with the assumption related to its elastic nature. Therefore, the necessity to take into consideration the physical non-linearities connected with the nature of the material itself may only be relevant for investigations of limit states of structures corresponding to loads significantly exceeding acceptable levels. The basic type of non-linearity taken into account in analyses of thin-walled aircraft structures is the geometrical non-linearity corresponding to the natural character of deformations in structures of this type (Felippa, 1976; Kopecki, 2010).

Verification of findings of the non-linear numerical analyses in terms of their credibility, in accordance with FEM, normally involves comparison of such findings with results of an experiment which can be conducted in a simplified way but has to properly map boundary conditions, geometrical proportions, and the nature of load affecting the part of the thin-walled structure in question (Doyle, 2001).

Results of a numerical analysis can be recognized as credible if the forms of post-critical deformations are fully consistent, and there is a satisfactory similarity in representative equilibrium paths constituting the relationships between a selected, representative parameter of the state determining the structure deformations and a representative control parameter connected with the load (Kopecki, 2010; Rakowski and Kacprzyk, 2005).

Credibility of results is assessed taking into account the principle of equivalent solutions in accordance with which one and only one stress condition corresponds to a given deformation state.

Consistency in the form and extent of deformation is crucial since there is no possibility to compare the actual equilibrium paths constituting the relationships between sets of control parameters and sets of state parameters. Therefore, the actual equilibrium path should be interpreted as a hypersurface within the hyperspace of states, satisfying a matrix equation of residual forces, which in the case of existing unambiguous relationship between load components takes the co-called monoparametric form

$$
\mathbf{r}(\mathbf{u}, \lambda)=\mathbf{0}
$$

where $\mathbf{u}$ is the vector of state comprising the components of displacements of the structure nodes corresponding to its current geometric configuration, $\lambda$ is the representative control parameter corresponding to the current load level, and $\mathbf{r}$ is the residual vector containing unbalanced components of forces which are connected with the current state of the system deformation. 
Methods of determining consecutive points of the equilibrium path employed in most commercial programs also contain a correction phase based on the satisfaction of an additional equation called the "equation of increment control" or "constraint equation" (Felippa, 1976)

$$
c\left(\Delta \mathbf{u}_{n}, \Delta \lambda_{n}\right)=0
$$

where the increments

$$
\Delta \mathbf{u}_{n}=\mathbf{u}_{n+1}-\mathbf{u}_{n} \quad \Delta \lambda_{n}=\lambda_{n+1}-\lambda_{n}
$$

correspond to the transition from state $n$ to $n+1$.

Non-linear numerical analysis of structural models in question has been carried out with the use of ABAQUS software. Calculations are based on the modified prognostic Newton-Raphson method combined with the standard load correction, and alternatively with the Crisfield hyperspherical correction (Bathe, 1996; Ramm, 1987) (Fig. 8).

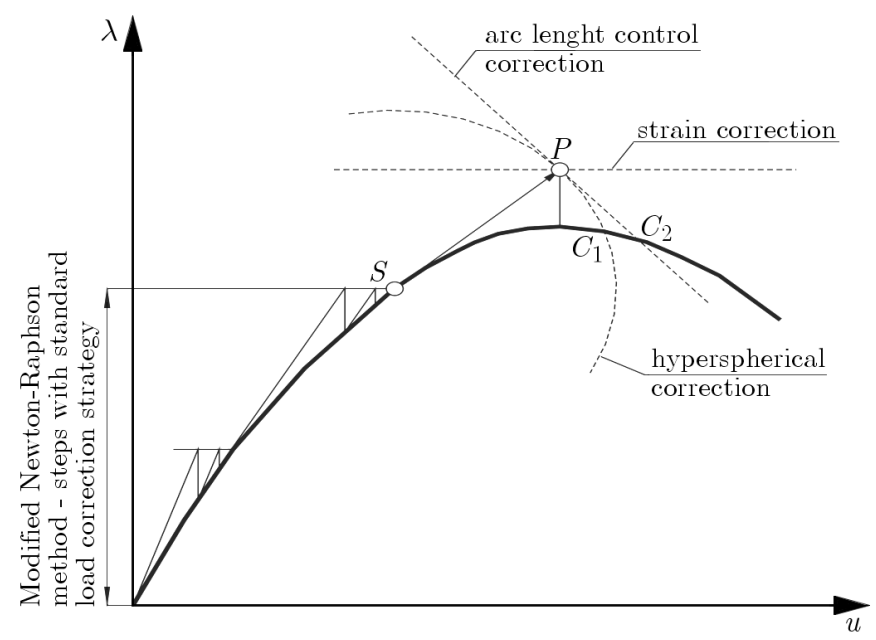

Fig. 8. Numerical methods employed in the non-linear FEM analysis

The numerical models take into account the fastening of the panels; this is done by mapping the frame elements and the connections between the frame and the panel via MPC elements of TIE type (Fig. 9).

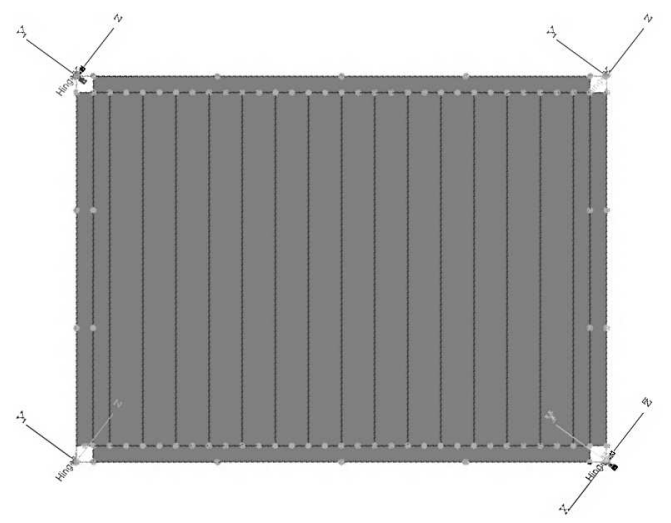

Fig. 9. Geometrical model of the integrally ribbed panel formed in the environment of Abaqus pre-processor

It has been shown by previous calculations that non-linear procedures employed in commercial programs are able to adequately determine forms of post-critical deformations in the 
case of smooth panels. Obtaining correct results in the case of integrally stiffened panels is a considerable challenge, and when model imperfections are not taken into consideration, this in fact becomes an impossible task. Due to the above, the first step for both types of structures involved conducting a simplified, linearized analysis of stability (linearized prebuckling). It has been aimed at obtaining a deformation in forms similar to those obtained during the experiment. These deformation forms, after appropriate calibration (reduction of the displacement rates along the direction normal to the panel surface down to the rates substantially lower than the structure dimensions) could be used as initial geometric imperfections during the proper non-linear analysis.

Ultimately, the findings of the analysis show the distribution of deformations (Fig. 10) which in a satisfactory way corresponds to the actual displacements measured during the experiment with the scanner. The similarity of the respective representative equilibrium paths is also deemed satisfactory.

(a)

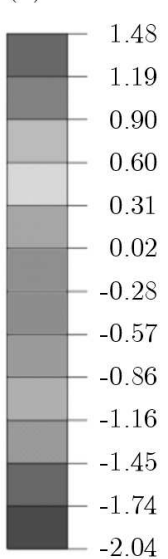

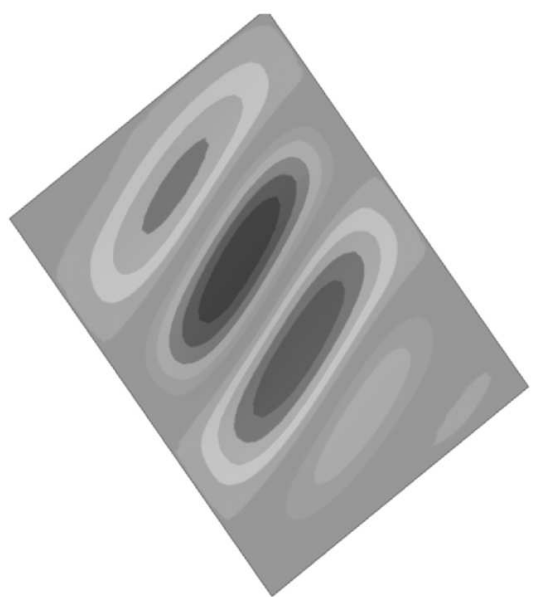

(b)

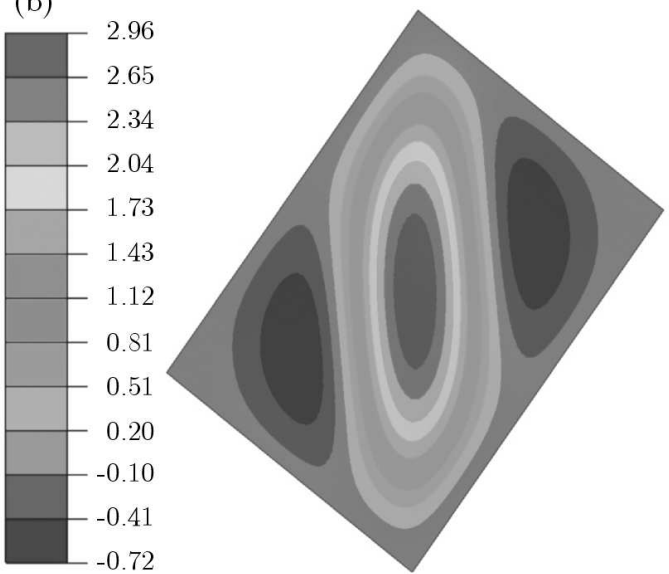

Fig. 10. Deformations determined via numerical method: (a) smooth panel, (b) ribbed panel
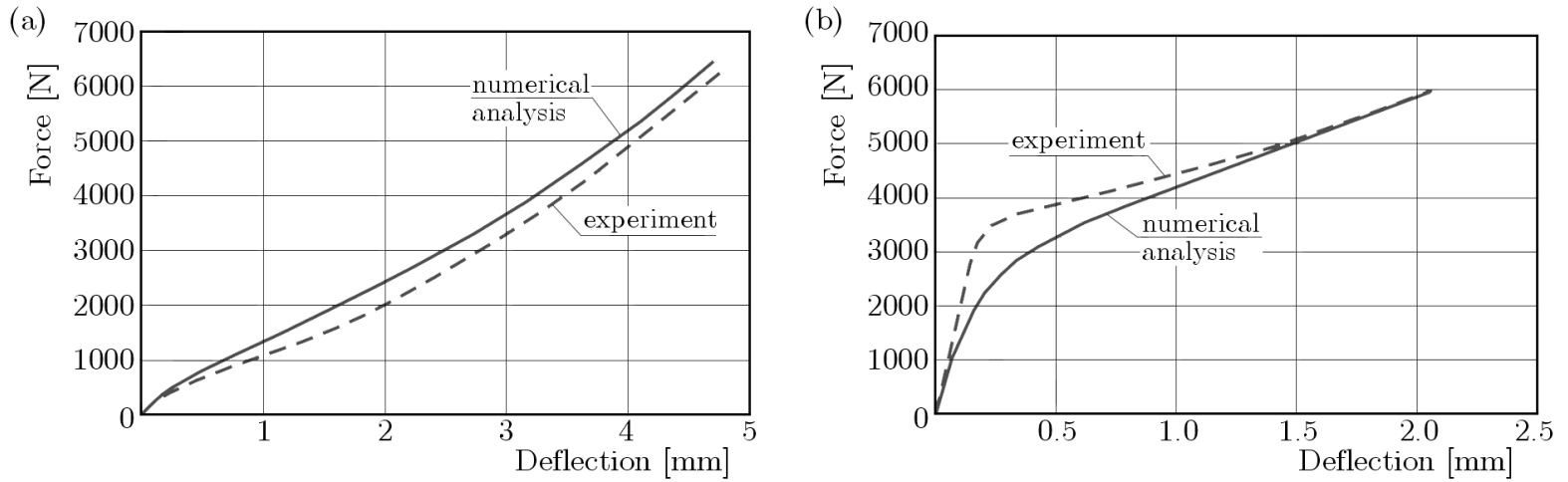

Fig. 11. Representative equilibrium paths determined via numerical method: (a) smooth panel, (b) ribbed panel

For the smooth panel, the similarity of the representative equilibrium paths is almost perfect. This proves that the mapping of the system stiffness, by introducing initial imperfections, is correct. The similarity of the characteristics in the post-critical range, in addition to the numerical mapping of the structure geometry and the boundary conditions, is also largely affected by the set of numerical methods, which themselves constitute elements of the algorithm of the non-linear procedure. In addition to prognostic methods and correction strategies, these programs include a whole series of ancillary procedures (e.g. algorithms for selecting branches of 
the equilibrium path in bifurcation points). In most commercial programs, the user may impact the selection and course of these procedures only to a very limited extent, and the obtained results are naturally encumbered with certain additional errors whose effects are difficult to define. Due to this, generally results of non-linear numerical analyses are not expected to be perfectly consistent with experimental results. The rate of acceptable error is determined depending on the software being used as well as the type of the analyzed structure and the designation of the component containing the structure. In most applications designed for learning purposes only, an incompatibility of results at the level of up to $10 \%$ is deemed to be completely satisfactory.

For the smooth panel in question, the rate of error for the maximum loads does not exceed $3 \%$, therefore the representative equilibrium paths can be deemed as fully compatible.

As far as the integrally stiffened panel is concerned, numerical mapping of initial geometry turned out to be encumbered with errors resulting from the differences between the form of deformation in the actual structure in an early phase of the experiment and the adopted geometric imperfections resulting from the findings of the simplified linearized stability analysis. However, it should be emphasized that in the advanced range of deformations corresponding to loads resembling maximum values, efficiency of the employed numerical procedures allow one to achieve almost perfect similarity of the representative equilibrium paths.

Therefore, it can be concluded that in accordance with the aforementioned principle of equivalent solutions the results obtained from numerical calculations satisfactorily correspond with the findings of the experiment, which shows that the adopted method of creating numerical models for actual structures is effective.

\section{Comparison of the properties of investigated models}

The above comparative analysis of the experimental findings and the non-linear numerical analyses provides the evidence confirming the credibility of the latter, with regard to both types of the structures in question.

Therefore, we can assume credibility of the reduced stress distributions according to Huber-Mises hypothesis (Fig. 12) resulting from the distributions of displacements obtained by means of the numerical method. It is likely that taking these into account, we can successfully compare the properties of both types of skins and their usefulness for load-bearing aircraft structures.

(a)

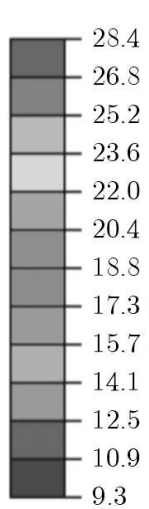

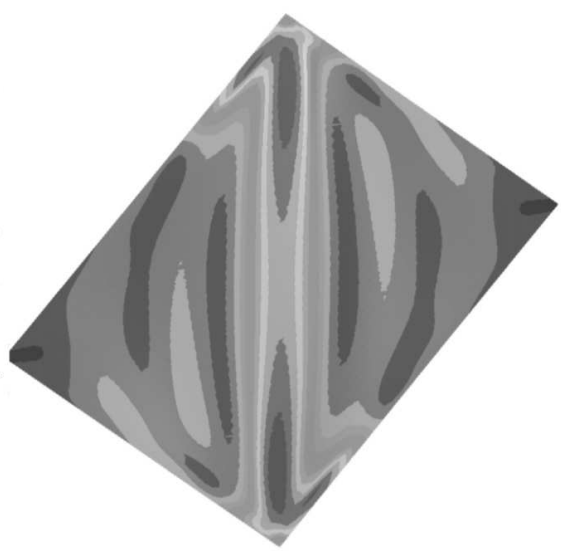

(b)

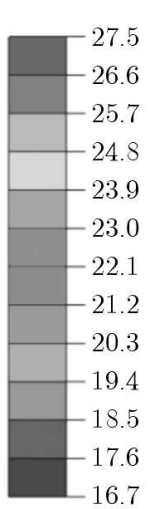

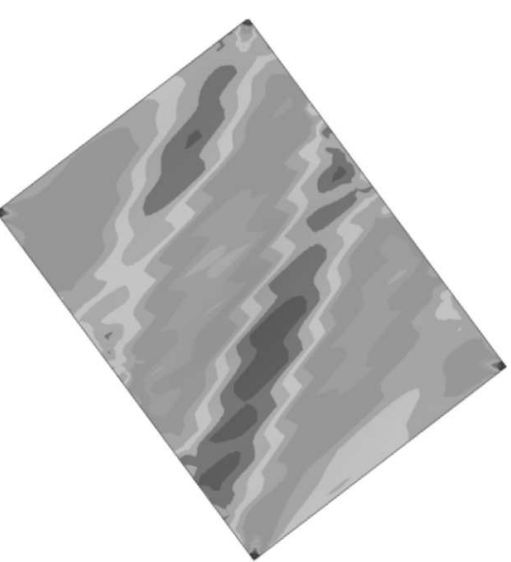

Fig. 12. Distribution of effort according to Huber-Mises hypothesis: (a) smooth panel, (b) ribbed panel

For the smooth panel, the distribution of effort is characteristic for structures of this type subjected to shearing and it can be interpreted as an effect of the occurring tension field. In this case, the function of the basic load-bearing element is taken over by certain fibres in the area 
of the panel diagonal. Those fibres are subjected to strong tension forces. Such a distribution is characterized by the presence of high gradients of reduced stress and strong concentrations near the panel corners. This leads to rapid loss of stability, and, as it is shown by statistics related to solutions of this type, to the occurrence of fatigue fractures after a relatively low number of load cycles.

In the case of the integrally ribbed panel, the nature of stress distribution seems to be substantially more favourable, due to both its gradients and the size of the structure area participating actively in the transfer of loads. In these two cases, the maximum stress level is comparable, yet the rate of the maximum gradient for the integrally ribbed panel is lower by $40 \%$ than for the smooth one. This suggests that the fatigue durability of the structure is substantially increased with the use of this solution.

An additional attribute of the integrally ribbed panel is the $75 \%$ increment of the critical load value. In operating conditions, this significantly reduces the exposition of the structure to cyclic work at post-critical deformations, which also contributes an increase in its operational durability.

\section{Summary and conclusions}

The presented considerations which mainly focus on comparing the structure comprising the solution investigated during this study with reference structure, additionally propose a method for carrying out relevant experiments as well as using the obtained results to specify numerical models in accordance with the finite element method.

The suggested method for verifying the results of non-linear numerical analysis by applying the principle of equivalent solutions seems to be effective, and the obtained results are sufficiently credible. This constituted the foundation for carrying out an initial comparative analysis of the physical properties of the load-bearing structures in question.

In the light of this analysis, the solution based on the use of integral ribs seems to be very promising from the point of view of its application in load-bearing aircraft structures. An unquestionable advantage of this solution is the relatively high level of critical load at low mass. This suggests potential application of such structures, e.g. as skins of working fragments in airframe wings where post-critical deformations contribute to the deterioration of the aerodynamic properties of the aircraft.

It is necessary to supplement the findings presented here, first of all with experimental analysis concerning the determination of bearing limit values and the impact of density and type of integral ribbing on the system stiffness. It is also recommended that the operational durability of the investigated structures should be determined through appropriate fatigue tests. It would also seem appropriate to perform a series of experiments with the use of models made of aluminium alloys used in the aviation industry.

\section{References}

1. Arborcz J., 1985, Post-buckling behavior of structures. Numerical techniques for more complicated structures, Lecture Notes in Physics, 228, USA

2. Bathe K.J., 1996, Finite Element Procedures, Prentice Hall, USA

3. Brzoska Z., 1965, Statics and Stability of Bar and Thin-Walled Structures, PWN, Warszawa, Poland

4. Doyle J.F., 2001, Nonlinear Analysis of Thin-Walled Structures, Springer-Verlag, Berlin, Germany 
5. Felippa C.A., 1976, Procedures for Computer Analysis of Large Nonlinear Structural System in Large Engineering Systems, A. Wexler (edit.), Pergamon Press, London, UK

6. Kopecki T., 2010, Advanced Deformation States in Thin-Walled Load-Bearing Structure Design Work, Publishing House of Rzeszów University of Technology, Rzeszów, Poland

7. Kopecki T., Mazurek P., 2013, Problems of numerical bifurcation reproducing in post-critical deformation states of aircraft structures, Journal of Theoretical and Applied Mechanics, 51, 4, 969-977

8. Kopecki T., Mazurek P., 2014, Numerical representation of post-critical deformations in the processes of determining stress distributions in closed multi-segment thin-walled aircraft load-bearing structures, Maintenance and Reliability, 16, 1, 164-169

9. Marcinowski J., 1999, Nonlinear Stability of Elastic Shells, Publishing House of Technical University of Wrocław, Poland

10. Niu M.C., 1988, Airframe Structural Design, Conmilit Press Ltd., Hong Kong

11. Rakowski G., Kacprzyk Z., 2005, Finite Elements Method in Structure Mechanics, Publishing House of Technical University of Warszawa, Warszawa, Poland

12. Ramm E., 1987, The Riks/Wempner Approach - An Extension of the Displacement Control Method in Nonlinear Analysis, Pineridge Press, Swensea, UK

Manuscript received March 6, 2013; accepted for print April 9, 2014 